\title{
Diabetes mellitus and extrapulmonary tuberculosis: site distribution and risk of mortality
}

\author{
M. J. MAGEE ${ }^{*}$, M. FOOTE 2 , S. M. RAY ${ }^{2}$, N. R. GANDHI ${ }^{2,3}$ AND \\ R. R. KEMPKER ${ }^{2}$ \\ ${ }^{1}$ Division of Epidemiology and Biostatistics, School of Public Health, Georgia State University, Atlanta, GA, \\ USA \\ ${ }^{2}$ Division of Infectious Diseases, Department of Medicine, Emory University School of Medicine, Atlanta, GA, \\ USA \\ ${ }^{3}$ Departments of Epidemiology and Global Health, Rollins School of Public Health, Emory University, Atlanta, \\ GA, USA
}

Received 23 September 2015; Final revision 8 January 2016; Accepted 5 February 2016;

first published online 1 March 2016

\section{SUMMARY}

Scarce data exist on the relationship between diabetes and extrapulmonary tuberculosis (EPTB). We evaluated whether diabetes impacts site of TB and risk of death in patients with EPTB. We evaluated a cohort of TB cases from the state of Georgia between 2009 and 2012. Patients aged $\geqslant 16$ years were classified by diabetes status according to medical records. Site of EPTB was determined by culture and/or state TB classification. Death was defined by all-cause mortality. Of 1325 eligible reported TB cases, $369(27 \cdot 8 \%)$ had any EPTB including $258(19 \cdot 5 \%)$ with only EPTB and $111(8 \cdot 4 \%)$ with pulmonary TB and EPTB. Of all TB cases, 158 had diabetes $(11.9 \%)$. In multivariable analysis, the odds of any EPTB was similar in patients with and without diabetes [adjusted odds ratio $1 \cdot 04,95 \%$ confidence interval (CI) $0 \cdot 70-1 \cdot 56$ ]. The risk of death was $23.8 \%$ in patients with EPTB and diabetes $v s .9 .8 \%$ in those with no diabetes $(P<0.01)$; after adjusting for covariates the difference was not significant (aRR $1 \cdot 19,95 \%$ CI $0 \cdot 54-2 \cdot 63$ ). Diabetes was common in patients with EPTB and risk of death was high. Improved understanding of the relationship between diabetes and EPTB is critical to determine the extent that diabetes affects TB diagnosis and clinical management.

Key words: Diabetes mellitus, extrapulmonary tuberculosis, tuberculosis.

\section{INTRODUCTION}

Globally, there are an estimated 9 million annual incident cases of active tuberculosis (TB) [1, 2]. The 2014 estimated worldwide prevalence of type 2 diabetes was $8 \cdot 3 \%$ (387 million persons) in adults, and is expected

\footnotetext{
* Author for correspondence: M. J. Magee, PhD, MPH, Division of Epidemiology and Biostatistics, School of Public Health, Georgia State University, PO Box 3984, Atlanta, GA 30302-3984, USA.

(Email: mjmagee@gsu.edu)
}

to surpass 590 million persons by 2035 [3]. The risk of active TB disease in patients with diabetes mellitus is estimated to be three times the risk of the general population and $15 \%$ of global active TB cases are attributable to diabetes [4-6]. Patients with diabetes may also have a greater risk of poor TB outcomes, including death and relapse [7]. Poor outcomes in patients with TB and diabetes may be due to immune impairment caused by diabetes $[8,9]$.

Nearly $15 \%$ of global TB cases are extrapulmonary tuberculosis (EPTB) cases. EPTB presents challenges 
to TB control because it is harder to diagnose [10] and certain forms are associated with worse outcomes [11]. Incidence of EPTB occurs more commonly in persons with impaired innate immunity [12, 13], renal disease [14], and HIV [15, 16]. Certain EPTB sites, including more severe sites such as central nervous system (CNS), are associated with HIV and lower CD4 cell count [17]. Although immune deficiency also occurs with diabetes, little is known about the epidemiological or clinical relationship between diabetes and EPTB.

Patients with diabetes are at increased risk of infections, including soft-tissue infections ( $S$. aureus, Candidia albicans) [18, 19], group B streptococcus [2022], and genitourinary infections (Enterobacteriaceae) $[23,24]$. The immune mechanisms that place patients with diabetes at increased risk of infections, including TB, may also increase the risk of EPTB.

If diabetes creates differential risk of EPTB, then this would have important implications for the clinical diagnosis and treatment of TB in patients with diabetes. Few studies have examined the relationship between diabetes and likelihood of EPTB. Moreover, even less is known regarding the risk of mortality in patients with EPTB and diabetes. Therefore, we aimed to determine the association between diabetes and EPTB and to determine the association between diabetes and risk of all-cause mortality during TB treatment in patients with EPTB.

\section{METHODS}

We conducted a retrospective observational study of all TB cases reported to the state of Georgia between January 2009 and September 2012 as described previously [25]. Eligible participants included all patients with pulmonary TB (PTB) or EPTB, aged $\geqslant 16$ years, and whose case was reported to the Georgia state TB register during the study period.

\section{Definitions}

The study outcomes of interest included site of TB disease and all-cause mortality. Site of TB disease was determined by culture and radiography and categorized as PTB, EPTB, or both combined. Further location of EPTB was determined using a combination of clinical diagnosis, radiography results, and specimen culture type. All-cause mortality was determined from patient medical records. Death from any cause occurring before or during TB treatment was defined as a death for this analysis.
The primary exposure of interest for all analyses was diabetes mellitus status. All patients were asked if they had ever been diagnosed with diabetes; patients who self-reported having diabetes were categorized as having diabetes. Additionally, medical records were reviewed and used to determine if diabetes status was previously diagnosed. As per standard of care, clinicians performed baseline blood chemistry tests, including random blood glucose, on all patients with $\mathrm{TB}$ and the results were also used to indicate diabetes status in the Georgia state TB register.

Patients' demographic and clinical characteristics were abstracted from the Georgia state TB registry and from medical records as described previously [25]. Key covariates of interest in this study included HIV status, foreign place of birth, and end-stage renal disease (ESRD).

\section{Data analyses}

We examined the association between diabetes status and site of TB, and the association between diabetes and risk of all-cause mortality. We used bivariate analyses $\left(\chi^{2}\right.$ test for categorical variables and KruskalWallis test for continuous variables) to determine the association between diabetes, patients' characteristics, and the outcome site of TB: pulmonary, extrapulmonary, or both combined. Logistic regression was used to report odds ratios (OR), adjusted odds ratios (aOR), and $95 \%$ confidence intervals $(\mathrm{CI})$ to compare the likelihood of having (1) EPTB-only, (2) both EPTB and PTB, or (3) any EPTB (patients with EPTB-only or both EPTB and PTB) by diabetes status and patients' clinical/demographic characteristics. For all three logistic models, the referent outcome comparison was to patients with PTB. To compare risk of all-cause mortality during EPTB treatment in patients with and without diabetes, we used log binomial regression to report risk ratios (RR), adjusted risk ratios (aRR), and $95 \%$ CI. Covariates included in regression models were chosen based on factors considered to be confounders (associated with both diabetes status and the models' outcome of interest) and using directed acyclic graph theory [26]. In multivariable analysis for the outcome site of TB, statistical interaction between diabetes and the covariates HIV, ESRD, and sex was assessed by including multiplicative terms in regression models [27]. For statistical tests, patients with HIV-positive results were compared to a composite group of all other patients (HIV negative and unknown HIV status). A two-sided $P$ value $\leqslant 0 \cdot 05$ was 
Table 1. Characteristics of patients with tuberculosis by pulmonary and extrapulmonary locations

\begin{tabular}{|c|c|c|c|c|}
\hline Characteristic & $\begin{array}{l}\text { Pulmonary } \\
N(\%) \\
956(72 \cdot 1)\end{array}$ & $\begin{array}{l}\text { Extrapulmonary } \\
N(\%) \\
258(19 \cdot 5)\end{array}$ & $\begin{array}{l}\text { Pulmonary and extrapulmonary } \\
N(\%) \\
111(8 \cdot 4)\end{array}$ & $P$ value* \\
\hline Diabetes mellitus & $116(12 \cdot 1)$ & $27(10 \cdot 5)$ & $15(13 \cdot 5)$ & 0.66 \\
\hline $\mathrm{HIV}_{\dagger}^{\dagger}$ & $95(9 \cdot 9)$ & $28(10 \cdot 9)$ & $31(27 \cdot 9)$ & $<0.01$ \\
\hline Sex (male) & $645(67 \cdot 5)$ & $157(60 \cdot 8)$ & $81(73 \cdot 0)$ & $0 \cdot 05$ \\
\hline Median age, years (IQR) & $46 \cdot 0(31 \cdot 0-57 \cdot 0)$ & $41 \cdot 5(30 \cdot 0-57 \cdot 0)$ & $44 \cdot 0(33-55 \cdot 0)$ & $0 \cdot 22$ \\
\hline \multicolumn{5}{|l|}{ Race/ethnicity } \\
\hline NH White & $160(16 \cdot 8)$ & $31(12 \cdot 0)$ & $13(11 \cdot 7)$ & \multirow[t]{4}{*}{0.08} \\
\hline NH Black & $444(46 \cdot 5)$ & $132(51 \cdot 2)$ & $63(56 \cdot 8)$ & \\
\hline NH Asian & $163(17 \cdot 1)$ & $55(21 \cdot 3)$ & $17(15 \cdot 3)$ & \\
\hline Hispanic & $187(19 \cdot 6)$ & $40(15 \cdot 5)$ & $18(16 \cdot 2)$ & \\
\hline Foreign born & $428(44 \cdot 9)$ & $122(47 \cdot 7)$ & $50(45 \cdot 0)$ & 0.73 \\
\hline Recent homelessness & $104(10 \cdot 9)$ & $11(4 \cdot 4)$ & $12(10 \cdot 9)$ & $<0.01$ \\
\hline Correctional facility: & $83(8 \cdot 7)$ & $15(5 \cdot 9)$ & $12(10 \cdot 9)$ & $0 \cdot 21$ \\
\hline Heavy alcohol use & $172(18 \cdot 2)$ & $14(5 \cdot 6)$ & $18(16 \cdot 4)$ & $<0.01$ \\
\hline Illicit drug use & $106(11 \cdot 2)$ & $12(4 \cdot 8)$ & $9(8 \cdot 2)$ & $<0.01$ \\
\hline ESRD & $18(1 \cdot 9)$ & $13(5 \cdot 0)$ & $4(3 \cdot 6)$ & $\mathbf{0 . 0 2}$ \\
\hline Previous TB treatment & $60(6 \cdot 3)$ & $12(4 \cdot 7)$ & $5(4 \cdot 5)$ & $0 \cdot 53$ \\
\hline \multicolumn{5}{|l|}{ DST profile } \\
\hline None to RIF/INH & $671(88 \cdot 6)$ & $129(91 \cdot 5)$ & $81(89 \cdot 0)$ & \multirow[t]{4}{*}{$0 \cdot 75$} \\
\hline RIF or INH & $81(10 \cdot 7)$ & $12(8 \cdot 5)$ & $9(9 \cdot 9)$ & \\
\hline Multidrug resistant & $5(0 \cdot 7)$ & 0 & $1(1 \cdot 1)$ & \\
\hline Unavailable & 199 & 117 & 20 & \\
\hline Death & $77(8 \cdot 0)$ & $28(10 \cdot 9)$ & $12(10 \cdot 8)$ & $0 \cdot 28$ \\
\hline
\end{tabular}

AFB, Acid fast bacilli; DST, drug susceptibility test; ESRD, end-stage renal disease; INH, isoniazid; IQR, interquartile range; $\mathrm{NH}$, non-Hispanic; RIF, rifampin.

* Two-sided $\chi^{2}$ test.

$\dagger$ HIV unknown categorized as negative.

$\$$ Diagnosed with TB in a correctional facility.

$\S$ All-cause mortality before or during or TB treatment.

Bold indicates statistically significant $P$ value $<0 \cdot 05$.

considered statistically significant throughout all analyses.

\section{Ethical review}

The study was approved by the Institutional Review Boards of Emory University and the Georgia Department of Public Health.

\section{RESULTS}

There were 1428 patients with active TB reported to the state of Georgia during the study period. A total of $1325(93 \%)$ patients with active TB were eligible for inclusion in the present study. Overall, patients were mostly male (67\%), US-born (55\%), and African American (48\%), with a median age of 45 years (interquartile range 31-57). HIV status was known for 1231 patients (93\%), 54 patients were not offered an HIV test, 22 refused, and 18 had missing information. Diabetes was prevalent in $11.4 \%$ of patients, $11 \cdot 1 \%$ had HIV, and $0.5 \%$ had both diabetes and HIV. EPTB was present in $369(27.8 \%)$ patients: $258(19.5 \%)$ had only EPTB and $111(8.4 \%)$ had both PTB and EPTB (Table 1). Of patients with any EPTB, common sites of infection included lymphatic $(n=102,27 \%)$, bone or joints $(n=31,8 \cdot 4 \%)$, CNS $(n=37,10 \%)$, and pleural $(n=100,27 \%)$ (Fig. 1). Of 42 patients with diabetes and any EPTB, lymphatic $(n=9,21 \%)$ and pleural $(n=14,33 \%)$ were the most common sites of infection (Supplementary Table 1). In addition, six (14\%) of those with diabetes and any EPTB had ESRD.

The prevalence of any EPTB-only in patients with diabetes was $26.6 \%$ compared to $28.0 \%$ in those without diabetes $(P=0 \cdot 66)$. The prevalence of EPTB-only in patients with diabetes was $17 \cdot 1 \%$ compared to $19.8 \%$ in those without diabetes and the prevalence 


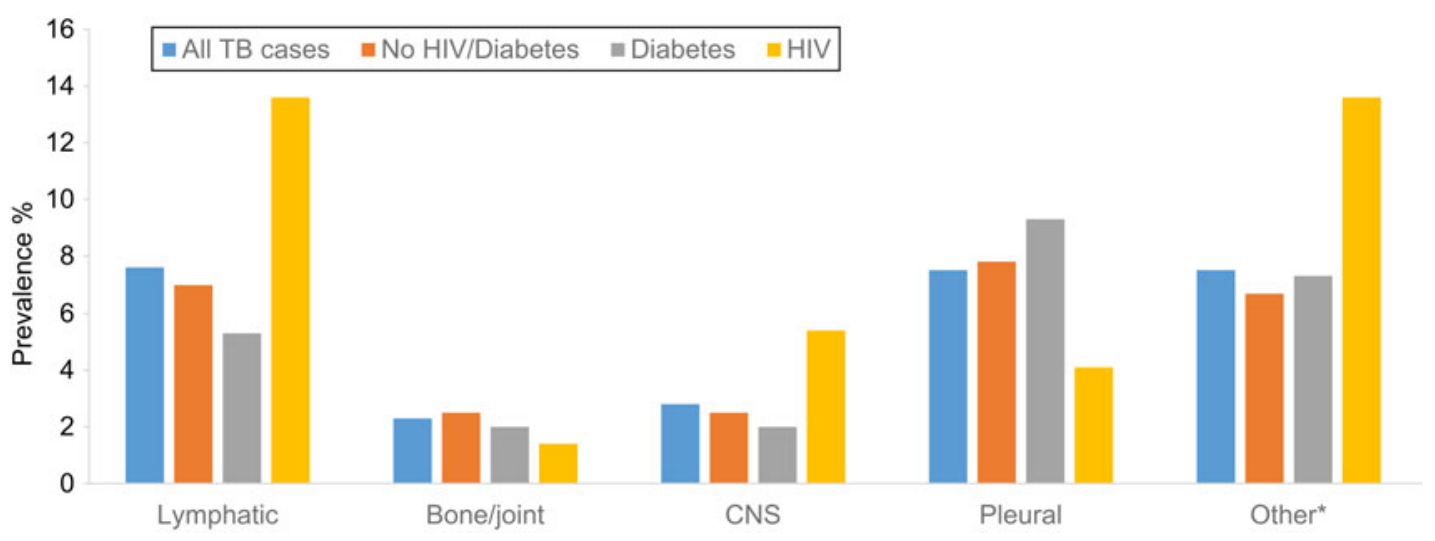

Fig. 1. Distribution of extrapulmonary tuberculosis sites of infection $(n=1325)$ * Other includes genitourinary, breast, gastrointestinal, peritoneal, cranial, pericardium, appendages.

Table 2. Patients' characteristics associated with having extrapulmonary site of tuberculosis

\begin{tabular}{|c|c|c|c|c|c|c|}
\hline \multirow[b]{2}{*}{ Characteristic } & \multicolumn{2}{|c|}{$\begin{array}{l}\text { EPTB-only vs. PTB } \\
(N=1214)\end{array}$} & \multicolumn{2}{|c|}{$\begin{array}{l}\text { EPTB and PTB-only vs. PTB } \\
(N=1067)\end{array}$} & \multicolumn{2}{|c|}{$\begin{array}{l}\text { Any EPTB } v s . \text { PTB } \\
(N=1325)\end{array}$} \\
\hline & OR $(95 \% \mathrm{CI})$ & aOR $(95 \% \mathrm{CI})$ & OR $(95 \% \mathrm{CI})$ & aOR $(95 \% \mathrm{CI})$ & OR $(95 \% \mathrm{CI})$ & $\mathrm{aOR}(95 \% \mathrm{CI})$ \\
\hline Diabetes mellitus* & $0 \cdot 85(0 \cdot 54-1 \cdot 32)$ & $0.92(0.57-1.47)$ & $1 \cdot 13(0 \cdot 64-2 \cdot 02)$ & $1 \cdot 39(0 \cdot 75-2 \cdot 58)$ & $0.93(0.64-1 \cdot 35)$ & $1 \cdot 04(0 \cdot 70-1 \cdot 56)$ \\
\hline $\mathrm{HIV}^{\dagger}$ & $1 \cdot 10(0 \cdot 71-1 \cdot 72)$ & $1 \cdot 15(0 \cdot 71-1 \cdot 87)$ & $3 \cdot 51(2 \cdot 21-5 \cdot 60)$ & $3 \cdot 46(2 \cdot 04-5 \cdot 85)$ & $1 \cdot 73(1 \cdot 22-2 \cdot 45)$ & $1 \cdot 82(1 \cdot 24-2 \cdot 68)$ \\
\hline \multicolumn{7}{|l|}{ Age, years } \\
\hline $16-34$ & $1 \cdot 00$ & $1 \cdot 00$ & $1 \cdot 00$ & $1 \cdot 00$ & $1 \cdot 00$ & $1 \cdot 00$ \\
\hline $35-44$ & $0 \cdot 89(0 \cdot 60-1 \cdot 33)$ & $0 \cdot 88(0 \cdot 58-1 \cdot 33)$ & $1 \cdot 76(1 \cdot 01-3 \cdot 08)$ & $1 \cdot 45(0 \cdot 81-2 \cdot 59)$ & $1.09(0 \cdot 77-1 \cdot 55)$ & $1 \cdot 03(0 \cdot 72-1 \cdot 48)$ \\
\hline $45-54$ & $0.57(0 \cdot 38-0 \cdot 84)$ & $0.59(0.38-0.90)$ & $1 \cdot 13(0 \cdot 65-1 \cdot 96)$ & $0.94(0.52-1 \cdot 70)$ & $0 \cdot 70(0 \cdot 50-0.98)$ & $0.68(0.47-0.97)$ \\
\hline$\geqslant 55$ & $0.74(0.52-1 \cdot 04)$ & $0 \cdot 72(0 \cdot 49-1 \cdot 05)$ & $0 \cdot 96(0 \cdot 56-1 \cdot 65)$ & $0.93(0.51-1.68)$ & $0 \cdot 79(0.58-1 \cdot 07)$ & $0 \cdot 78(0 \cdot 56-1 \cdot 10)$ \\
\hline Sex (female) & $1 \cdot 33(1 \cdot 00-1 \cdot 77)$ & $1 \cdot 22(0 \cdot 91-1 \cdot 64)$ & $0 \cdot 77(0 \cdot 50-1 \cdot 19)$ & $0 \cdot 75(0 \cdot 47-1 \cdot 18)$ & $1 \cdot 14(0 \cdot 89-1 \cdot 47)$ & $1 \cdot 08(0 \cdot 83-1 \cdot 40)$ \\
\hline \multicolumn{7}{|c|}{, } \\
\hline NH White & $1 \cdot 00$ & $1 \cdot 00$ & $1 \cdot 00$ & $1 \cdot 00$ & $1 \cdot 00$ & $1 \cdot 00$ \\
\hline NH Black & $1 \cdot 53(1 \cdot 00-2 \cdot 36)$ & $1 \cdot 40(0 \cdot 89-2 \cdot 19)$ & $1 \cdot 75(0 \cdot 94-3 \cdot 26)$ & $1 \cdot 32(0 \cdot 69-2 \cdot 52)$ & $1 \cdot 60(1 \cdot 10-2 \cdot 32)$ & $1 \cdot 39(0 \cdot 95-2 \cdot 05)$ \\
\hline NH Asian & $1 \cdot 74(1 \cdot 07-2 \cdot 85)$ & $1 \cdot 41(0 \cdot 85-2 \cdot 35)$ & $1 \cdot 28(0 \cdot 60-2 \cdot 73)$ & $1 \cdot 21(0 \cdot 56-2 \cdot 63)$ & $1 \cdot 61(1 \cdot 04-2 \cdot 48)$ & $1 \cdot 40(0 \cdot 90-2 \cdot 19)$ \\
\hline Hispanic & $1 \cdot 10(0 \cdot 66-1 \cdot 85)$ & $0.94(0.54-1.62)$ & $1 \cdot 19(0 \cdot 56-2 \cdot 49)$ & $0.96(0.44-2 \cdot 12)$ & $1 \cdot 13(0 \cdot 72-1 \cdot 76)$ & $0.97(0 \cdot 60-1 \cdot 55)$ \\
\hline $\begin{array}{l}\text { Recent } \\
\text { homelessness }\end{array}$ & $0 \cdot 37(0 \cdot 19-0 \cdot 69)$ & $0 \cdot 41(0 \cdot 21-0 \cdot 80)$ & $0 \cdot 99(0 \cdot 53-1 \cdot 87)$ & $0 \cdot 60(0 \cdot 30-1 \cdot 21)$ & $0 \cdot 55(0 \cdot 35-0 \cdot 87)$ & $0 \cdot 49(0 \cdot 30-0 \cdot 81)$ \\
\hline ESRD & $2 \cdot 77(1 \cdot 34-5 \cdot 72)$ & $2 \cdot 67(1 \cdot 25-5 \cdot 71)$ & $1 \cdot 95(0 \cdot 65-5 \cdot 86)$ & $1.59(0.51-4.99)$ & $2 \cdot 52(1 \cdot 28-4 \cdot 94)$ & $2 \cdot 27(1 \cdot 13-4 \cdot 57)$ \\
\hline
\end{tabular}

aOR, Adjusted odds ratio; CI, confidence interval; EPTB, extrapulmonary tuberculosis; ESRD, end-stage renal disease; $\mathrm{NH}$, non-Hispanic; OR, odds ratio; PTB, pulmonary tuberculosis.

* Multivariable model adjusted for age, sex, race/ethnicity, HIV, ESRD, recent homelessness.

$\dagger$ HIV unknown categorized as negative. Multivariable model adjusted for age, sex, race/ethnicity, ESRD, recent homelessness.

of PTB and EPTB-only was $9 \cdot 5 \%$ (diabetes) and $8 \cdot 2 \%$ (no diabetes). Only ESRD, HIV, and male sex were significantly associated with EPTB. In multivariable analysis, patients with diabetes, compared to patients without diabetes, were non-significantly more likely to have both PTB and EPTB (aOR 1.39, 95\% CI 0.752.58) after adjusting for age, sex, race/ethnicity, HIV status, ESRD and recent homelessness (Table 2).

Of 369 patients with any EPTB, 42 (11\%) died including $31 \%(13 / 42)$ before TB treatment initiation
(Table 3). The unadjusted risk of death in EPTB patients with diabetes was $2 \cdot 43$ (95\% CI 1.29-4.58). After adjusting for sex, age, foreign-born status, HIV, EPTB site, and ESRD, the adjusted risk of mortality in patients with diabetes was $1 \cdot 19(95 \%$ CI 0.54 2.63) times the risk in patients without diabetes. Factors significantly associated with mortality included HIV (aRR $2 \cdot 18$, 95\% CI 1·06-4.46), CNS site of infection (aRR $3 \cdot 79,95 \%$ CI $1 \cdot 32-8 \cdot 47$ ), and ESRD (aRR 3·48, 95\% CI 1·43-8·47). 
Table 3. Risk factors for mortality during tuberculosis treatment in patients with EPTB

\begin{tabular}{|c|c|c|c|}
\hline Characteristic & $\begin{array}{l}\text { Mortality risk } \\
42 / 369(11 \cdot 4 \%) \\
\operatorname{Died} / N(\%)\end{array}$ & $\begin{array}{l}\text { Crude RR } \\
(95 \% \mathrm{CI})\end{array}$ & $\begin{array}{l}\text { Adjusted RR* } \\
(95 \% \mathrm{CI})\end{array}$ \\
\hline \multicolumn{4}{|c|}{ Diabetes mellitus } \\
\hline No & $32 / 327(9 \cdot 8)$ & $1 \cdot 00$ & $1 \cdot 00$ \\
\hline Yes & $10 / 42(23 \cdot 8)$ & $2 \cdot 43(1 \cdot 29-4 \cdot 58)$ & $1 \cdot 19(0 \cdot 54-2 \cdot 63)$ \\
\hline \multicolumn{4}{|l|}{$\mathrm{HIV} \dagger$} \\
\hline No & $30 / 310(9 \cdot 7)$ & $1 \cdot 00$ & $1 \cdot 00$ \\
\hline Yes & $12 / 59(20 \cdot 3)$ & $2 \cdot 10(1 \cdot 14-3 \cdot 86)$ & $2 \cdot 18(1 \cdot 06-4 \cdot 46)$ \\
\hline \multicolumn{4}{|l|}{ EPTB site } \\
\hline Lymphatic & $7 / 102(6 \cdot 9)$ & $1 \cdot 00$ & $1 \cdot 00$ \\
\hline Bone/joint & $3 / 31(9 \cdot 7)$ & $1 \cdot 41(0 \cdot 39-5 \cdot 13)$ & \\
\hline $\mathrm{CNS}$ & $10 / 37(27 \cdot 0)$ & $3.94(1.62-9.59)$ & $3.79(1.42-8.47)$ \\
\hline Pleural & $11 / 100(11 \cdot 0)$ & $1.60(0.65-3 \cdot 97)$ & \\
\hline Other§ & $11 / 99(11 \cdot 1)$ & $1 \cdot 61(0 \cdot 65-4 \cdot 01)$ & \\
\hline \multicolumn{4}{|l|}{ Sex } \\
\hline Female & $10 / 131(7 \cdot 6)$ & $1 \cdot 00$ & $1 \cdot 00$ \\
\hline Male & $32 / 238(13 \cdot 5)$ & $1 \cdot 76(0 \cdot 89-3 \cdot 47)$ & $1 \cdot 53(0 \cdot 75-3 \cdot 15)$ \\
\hline \multicolumn{4}{|l|}{ Age, years } \\
\hline $16-34$ & $6 / 129(4 \cdot 7)$ & $1 \cdot 00$ & $1 \cdot 00$ \\
\hline $35-44$ & $4 / 72(5 \cdot 6)$ & $1 \cdot 19(0 \cdot 35-4 \cdot 09)$ & \\
\hline $45-54$ & $10 / 69(14 \cdot 5)$ & $3 \cdot 12(1 \cdot 18-8 \cdot 21)$ & $4 \cdot 77(2 \cdot 16-10 \cdot 51) \|$ \\
\hline$\geqslant 55$ & $22 / 99(22 \cdot 2)$ & $4 \cdot 78(2 \cdot 01-11 \cdot 33)$ & \\
\hline \multicolumn{4}{|l|}{ Race/ethnicity } \\
\hline NH White & $9 / 44(20 \cdot 5)$ & $1 \cdot 00$ & \\
\hline NH Black & $27 / 195(13 \cdot 9)$ & $0 \cdot 68(0 \cdot 34-1 \cdot 34)$ & \\
\hline NH Asian & $2 / 72(2 \cdot 8)$ & $0 \cdot 14(0 \cdot 03-0 \cdot 60)$ & \\
\hline Hispanic & $4 / 58(6 \cdot 9)$ & $0 \cdot 34(0 \cdot 11-1 \cdot 02)$ & \\
\hline \multicolumn{4}{|l|}{ Foreign born } \\
\hline No & $32 / 197(16 \cdot 2)$ & $1 \cdot 00$ & $1 \cdot 00$ \\
\hline Yes & $10 / 172(5 \cdot 8)$ & $0 \cdot 36(0 \cdot 18-0 \cdot 71)$ & $0 \cdot 72(0 \cdot 34-1 \cdot 53)$ \\
\hline \multicolumn{4}{|c|}{ Recent homelessness } \\
\hline No & $38 / 346(11 \cdot 0)$ & $1 \cdot 00$ & \\
\hline Yes & $4 / 23(17 \cdot 4)$ & $1 \cdot 58(0 \cdot 62-4 \cdot 05)$ & \\
\hline \multicolumn{4}{|c|}{ Correctional facility\# } \\
\hline No & $42 / 342(12 \cdot 3)$ & $1 \cdot 00$ & \\
\hline Yes & $0 / 27(0)$ & n.a. & \\
\hline \multicolumn{4}{|c|}{ Heavy alcohol use } \\
\hline No & $35 / 302(10 \cdot 4)$ & $1 \cdot 00$ & \\
\hline Yes & $7 / 32(21 \cdot 9)$ & $2 \cdot 11(1 \cdot 02-4 \cdot 35)$ & \\
\hline \multicolumn{4}{|l|}{ Illicit drug use } \\
\hline No & $40 / 348(11 \cdot 5)$ & $1 \cdot 00$ & \\
\hline Yes & $2 / 21(9 \cdot 5)$ & $0 \cdot 83(0 \cdot 21-3 \cdot 20)$ & \\
\hline \multicolumn{4}{|l|}{ ESRD } \\
\hline No & $35 / 352(9 \cdot 9)$ & $1 \cdot 00$ & $1 \cdot 00$ \\
\hline Yes & $7 / 17(41 \cdot 2)$ & $4 \cdot 14(2 \cdot 16-7 \cdot 92)$ & $3 \cdot 48(1 \cdot 43-8 \cdot 47)$ \\
\hline \multicolumn{4}{|c|}{ Previous TB treatment } \\
\hline No & $39 / 352(11 \cdot 1)$ & $1 \cdot 00$ & \\
\hline Yes & $3 / 17(17 \cdot 7)$ & $1.59(0.55-4 \cdot 64)$ & \\
\hline
\end{tabular}

CNS, Central nervous system; CI, confidence interval; EPTB, extrapulmonary tuberculosis; ESRD, end-stage renal disease, $\mathrm{NH}$, non-Hispanic; RR, risk ratio.

* Multivariable model included diabetes mellitus, HIV, EPTB site, sex, age, foreign born, and ESRD.

$\dagger$ HIV unknown categorized as negative.

$\$$ In multivariable model, EPTB site was dichotomous as CNS vs. all other.

$\S$ Other includes genitourinary, breast, gastrointestinal, peritoneal, cranial, pericardium, appendages.

|| In multivariable model, age was dichotomous as $<45$ and $\geqslant 45$ years.

\# TB diagnosed in a correctional facility.

Bold indicates statistically significant, $P$ value $<0 \cdot 05$. 
In an assessment of statistical interaction, the effect of diabetes on prevalence of any EPTB was not significantly different by HIV, ESRD, or sex. However, there was a non-significant trend $(P=0 \cdot 42)$ for increased prevalence of EPTB in those with diabetes and ESRD (aOR 4.22, 95\% CI 1·11-16.13) compared to those with-

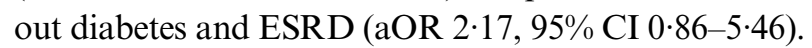
Similarly the effect of diabetes on risk of death in patients with EPTB was not significantly different by HIV, ESRD, or site of extrapulmonary infection.

\section{DISCUSSION}

In this retrospective cohort of adult patients with any EPTB from the state of Georgia, diabetes was common $(11.4 \%)$ and lymphatic and pleural sites of infection were the most frequent sites of extrapulmonary disease in patients with diabetes and EPTB. Of patients with EPTB-only, the prevalence of diabetes $(10 \cdot 5 \%)$ was also high, and similar compared to the prevalence of HIV (10.9\%). We did not find that patients with diabetes were more likely to have EPTB compared to PTB. We did observe a high proportion $(23.8 \%)$ of all-cause mortality in patients with EPTB and diabetes, although the observed crude association between diabetes and risk of death was largely due to other patient factors associated with death in those with diabetes, most notably age and ESRD. Our study also confirmed previous findings that HIV is associated with EPTB [1] and an increased risk of death in patients with EPTB [11], and we reported ESRD to be associated with both EPTB and increased risk of death.

Few previous studies to date have examined the association between diabetes and EPTB. One population-based study of adult TB patients $(n=$ 1194) in Galicia, Spain, reported a non-significant increased odds of having EPTB vs. PTB in patients with diabetes (OR 1.48, 95\% CI 0.89-2.47) [28]. Another study analysing TB cases during 20072011 from Brazil's Notifiable Disease Surveillance System reported that $8.4 \%$ of patients with diabetes had EPTB compared to $14.7 \%$ in those without diabetes $(P<0 \cdot 01)$ [29]. The multivariable model from the Brazilian analysis only adjusted for clinical characteristics and did not aim to estimate the association between diabetes and EPTB. Third, a Taiwanese hospital-based study of 766 patients with TB reported that diabetes was associated with decreased likelihood of EPTB (aOR $0 \cdot 41,95 \%$ CI $0 \cdot 22-0 \cdot 76$ ) [14]. However, the Taiwanese study used stepwise regression methods and likely adjusted for factors on the causal pathway between diabetes and risk of TB leading to a biased effect of diabetes on risk of EPTB. Considering our results in the context of previous studies, the majority of existing data suggest that diabetes likely does not increase the risk of EPTB. Nonetheless, the global prevalence of diabetes will continue to increase substantially in the next 20 years, and given the paucity of studies that were specifically designed to examine the relationship between diabetes and EPTB, more investigations will be needed. Future diabetes EPTB studies will likely be improved by Xpert assay technology which has recently been demonstrated to be a sensitive and specific tool to detect and diagnose EPTB [10].

The high all-cause mortality rate $(11 \cdot 4 \%)$ in patients with EPTB observed in this study is also consistent with previous reports of mortality in patients with EPTB in the state of Georgia. For example, in a study conducted during 1995-2001, Kourbatova et al. reported the 12-month mortality rate in patients with EPTB at an inner-city Atlanta hospital was $14 \cdot 6 \%$ [11]. In our study the unadjusted risk of death in patients with EPTB and diabetes was more than twice the risk in those without diabetes; however, this apparent increased risk of death was likely confounded by age and ESRD. In this study, age and ESRD were associated with both diabetes and with increased risk of death, after adjusting for age, ESRD, and other factors, the estimated effect of diabetes on risk of death was substantially attenuated (RR $1 \cdot 19,95 \%$ CI $0 \cdot 54-2 \cdot 63$ ).

We did not detect significant statistical interaction between diabetes and HIV, ESRD, or sex. However, the finding that patients with both ESRD and diabetes had an increased trend towards any EPTB (aOR 4.22 95\% CI 1.11-16.13) suggests there may be synergy between ESRD and diabetes with the risk of EPTB. Chronic renal disease is a risk factor for active TB [30] and previous studies reported a high prevalence of EPTB in patients on dialysis [31] and ESRD [14]. Consistent with our findings that ESRD is associated with more than twice the odds of EPTB, a study in Taiwan reported that the odds of EPTB was significantly higher in patients with ESRD (aOR 3.74, 95\% CI 1.45-9.67) [14]. Kidney damage may lead to an increased risk of developing EPTB in part due to latent $\mathrm{TB}$ infection existing in kidneys. A recent study of 49 necropsy specimens from Mexican patients who died from causes other than TB found that 43 had mycobacterical DNA in EPTB sites, including $79 \%(34 / 43)$ with mycobacterial DNA in the kidneys 
[32]. Globally, the number of patients receiving renal replacement therapy doubled during 1990-2010 and is expected to double again by 2030 , reaching 5.4 million people [33]. Given the aetiological connection between diabetes and ESRD, and the marked expected increases in both diseases, an improved understanding of the diabetes-ESRD-TB epidemiological relationships are needed. Specifically, whether the risk of EPTB in patients with ESRD is modified by diabetes should be evaluated in future studies.

Although not the primary focus of our study, we also found that ESRD and HIV were significantly associated with all-cause mortality, even after adjusting for multiple patient clinical characteristics. To our knowledge, our study is the first to report that ESRD is associated with all-cause mortality in patients with EPTB even after adjusting for key confounders such as age, HIV, and diabetes. Uraemia from ESRD is associated with persistent immune inflammation and reduced T-cell activation which may also contribute to increased mortality [34]. We previously reported HIV to be associated with CNS site of infection in patients with EPTB [17], and CNS site of infection is associated with increased risk of mortality [11]. Unlike this study that reports HIV to be significantly associated with all-cause mortality in adjusted analyses, Kourbatova et al. reported that HIV was associated with mortality only in unadjusted analysis [11].

This study is subject to some limitations. First, we did not systematically screen all patients in this study for diabetes using haemoglobin A1c or another widely accepted diagnostic technique to measure diabetes. Instead we relied on medical record abstraction and self-reported diabetes status, consequently our measure of diabetes was subject to misclassification. Nonetheless, all patients did receive general blood chemistry tests and therefore patients with high glucose levels would likely be identified as having diabetes. In addition, self-reported diabetes status has been found to have high specificity, in our study patients were probably not falsely misclassified as having diabetes. Second, our study was unable to assess the relationship between glucose control, duration of diabetes, or immune function with EPTB. While we did not observe an association between diabetes and EPTB or site of EPTB, patients with poorly controlled diabetes may have greater immune impairment and more likely to have certain sites of EPTB; we were unable to examine this association. Third, specimen cultures were not available for all patients with EPTB, therefore classification of EPTB and site of EPTB may be misclassified. Nonetheless, we performed a subgroup analysis in only patients who had a positive EPTB specimen culture (data not shown). The magnitude, direction, and statistical significance of the subgroup analyses was the same as reported in our primary analyses.

\section{CONCLUSION}

The increasing global prevalence of diabetes has important implications for public health management of TB. Improved understanding of the relationship between diabetes and risk of EPTB is also critical to characterize the extent to which diabetes will affect TB diagnosis and clinical management. Our study thoroughly examined the relationship between diabetes and EPTB and found that diabetes does not significantly increase the likelihood of EPTB. Nonetheless, as incident active TB cases continue to decrease in the United States, a larger proportion of new TB cases in the United States will arise due to reactivation $[35,36]$ and consequently a greater proportion due to diabetes. If a larger proportion of TB cases in the United States are attributable to diabetes, the relationship between diabetes and EPTB will require re-examination.

\section{SUPPLEMENTARY MATERIAL}

For supplementary material accompanying this paper visit http://dx.doi.org/10.1017/S0950268816000364.

\section{ACKNOWLEDGEMENTS}

This work was supported in part by the National Institutes of Health (NIH) National Institute of Allergy and Infectious Diseases [K23AI103044 (R.R. K.) and K24AI114444 (N.R.G.)], Bethesda, MD, USA, the Emory Centers for AIDS Research (P30 AI050409) and the Emory University Laney Graduate School, Atlanta, GA, USA. The funders had no role in study design, data collection and analysis, decision to publish, or preparation of the manuscript.

\section{DECLARATION OF INTEREST}

None. 


\section{REFERENCES}

1. Peto HM, et al. Epidemiology of extrapulmonary tuberculosis in the United States, 1993-2006. Clinical Infectious Diseases 2009; 49: 1350-1357.

2. WHO. Global tuberculosis report 2014. Geneva: World Health Organization, 2014.

3. IDF. Diabetes Atlas, 6th edn. Brussels: International Diabetes Federation, 2013.

4. Jeon CY, Murray MB. Diabetes mellitus increases the risk of active tuberculosis: a systematic review of $13 \mathrm{ob}-$ servational studies. PLoS Medicine 2008; 5: e152.

5. Baker MA, et al. The risk of tuberculosis disease among persons with diabetes mellitus: a prospective cohort study. Clinical Infectious Diseases 2012; 54: 818-825.

6. Lonnroth K, Roglic G, Harries AD. Improving tuberculosis prevention and care through addressing the global diabetes epidemic: from evidence to policy and practice. Lancet Diabetes \& Endocrinology 2014; 2: 730-739.

7. Baker MA, et al. The impact of diabetes on tuberculosis treatment outcomes: a systematic review. BMC Medicine 2011; 9: 81 .

8. Martens GW, et al. Tuberculosis susceptibility of diabetic mice. American Journal of Respiratory Cell and Molecular Biology 2007; 37: 518-524.

9. Kumar NP, et al. Expansion of pathogen-specific T-helper 1 and T-helper 17 cells in pulmonary tuberculosis with coincident type 2 diabetes mellitus. Journal of Infectious Diseases 2013; 208: 739-748.

10. Denkinger CM, et al. Xpert MTB/RIF assay for the diagnosis of extrapulmonary tuberculosis: a systematic review and meta-analysis. European Respiratory Journal 2014; 44: 435-446.

11. Kourbatova $\mathbf{E V}$, et al. Risk factors for mortality among patients with extrapulmonary tuberculosis at an academic inner-city hospital in the US. European Journal of Epidemiology 2006; 21: 715-721.

12. Sterling TR, et al. Human immunodeficiency virusseronegative adults with extrapulmonary tuberculosis have abnormal innate immune responses. Clinical Infectious Diseases 2001; 33: 976-982.

13. Antas PR, et al. Decreased CD4+ lymphocytes and innate immune responses in adults with previous extrapulmonary tuberculosis. Journal of Allergy and Clinical Immunology 2006; 117: 916-923.

14. Lin $\mathbf{J N}$, et al. Risk factors for extra-pulmonary tuberculosis compared to pulmonary tuberculosis. International Journal of Tuberculosis and Lung Disease 2009; 13: 620625.

15. Yang Z, et al. Identification of risk factors for extrapulmonary tuberculosis. Clinical Infectious Diseases 2004; 38: 199-205.

16. Jones BE, et al. Relationship of the manifestations of tuberculosis to CD4 cell counts in patients with human immunodeficiency virus infection. American Review of Respiratory Disease 1993; 148: 1292-1297.

17. Leeds IL, et al. Site of extrapulmonary tuberculosis is associated with HIV infection. Clinical Infectious Diseases 2012; 55: 75-81.
18. Muller LM, et al. Increased risk of common infections in patients with type 1 and type 2 diabetes mellitus. Clinical Infectious Diseases 2005; 41: 281-288.

19. Peleg AY, et al. Common infections in diabetes: pathogenesis, management and relationship to glycaemic control. Diabetes/Metabolism Research and Reviews 2007; 23: 3-13.

20. Skoff TH, et al. Increasing burden of invasive group B streptococcal disease in nonpregnant adults, 1990 2007. Clinical Infectious Diseases 2009; 49: 85-92.

21. Farley MM. Group B streptococcal disease in nonpregnant adults. Clinical Infectious Diseases 2001; 33: 556-561.

22. Thomsen RW, et al. Impact of diabetes and poor glycaemic control on risk of bacteraemia with haemolytic streptococci groups A, B, and G. Journal of Infection 2011; 63: 8-16.

23. Ishay A, Lavi I, Luboshitzky R. Prevalence and risk factors for asymptomatic bacteriuria in women with Type 2 diabetes mellitus. Diabetic Medicine 2006; 23: 185-188.

24. Stapleton A. Urinary tract infections in patients with diabetes. American Journal of Medicine 2002; 113 (Suppl. 1A): 80S-84S.

25. Magee MJ, et al. Diabetes mellitus and risk of all-cause mortality among patients with tuberculosis in the state of Georgia, 2009-2012. Annals of Epidemiology 2014; 24: 369-375.

26. Greenland S, Pearl J, Robins JM. Causal diagrams for epidemiologic research. Epidemiology 1999; 10: 37-48.

27. Kleinbaum D, Klein M. Logistic Regression: A SelfLearning Text, Third Edition. New York, NY: Springer Science, 2010.

28. Garcia-Rodriguez JF, et al. Extrapulmonary tuberculosis: epidemiology and risk factors. Enfermedades Infecciosas y Microbiologia Clinica 2011; 29: 502-509.

29. Gomes T, et al. Epidemiology of extrapulmonary tuberculosis in Brazil: a hierarchical model. BMC Infectious Diseases 2014; 14: 9.

30. Hussein MM, Mooij JM, Roujouleh H. Tuberculosis and chronic renal disease. Seminars in Dialysis 2003; 16: $38-44$.

31. Lui SL, et al. Tuberculosis infection in Chinese patients undergoing continuous ambulatory peritoneal dialysis. American Journal of Kidney Diseases 2001; 38: 1055 1060.

32. Barrios-Payan J, et al. Extrapulmonary locations of mycobacterium tuberculosis DNA during latent infection. Journal of Infectious Diseases 2012; 206: 1194-1205.

33. Liyanage $\mathbf{T}$, et al. Worldwide access to treatment for end-stage kidney disease: a systematic review. Lancet 2015; 385: 1975-1982.

34. Meijers RW, et al. T-cell ageing in end-stage renal disease patients: Assessment and clinical relevance. World Journal of Nephrology 2014; 3: 268-276.

35. Bock NN, et al. A tuberculin screening and isoniazid preventive therapy program in an inner-city population. American Journal of Respiratory and Critical Care Medicine 1999; 159: 295-300.

36. Styblo K. Recent advances in epidemiological research in tuberculosis. Advances in Tuberculosis Research 1980; 20: $1-63$ 